\title{
OBITUARIO
}

\section{IN MEMORIAM ÁLVARO MATUTE AGUIRRE} (1943-2017)

\author{
Leonardo Lomelí Vanegas \\ Universidad Nacional Autónoma de México
}

F 112 de septiembre de 2017 falleció sorpresivamente en la Ciudad de México el doctor Álvaro Matute Aguirre. No fue fácil asimilar la pérdida de una persona tan querida y que se encontraba en plena madurez académica e intelectual. Tuvo una muerte apacible y tranquila, acorde a su personalidad. Se fue unos meses antes de recibir un reconocimiento por 50 años de antigüedad académica en la Universidad Nacional Autónoma de México y de formalizar su ingreso a la Academia Mexicana de la Lengua.

Cuando uno revisa su biografía académica e intelectual, cuesta trabajo imaginar al niño que hace setenta años perdió a uno de sus afectos más entrañables y que representó su primer contacto íntimo con la historia de la revolución mexicana: su abuelo, el general e ingeniero Amado Aguirre, quien acompañó a Álvaro Obregón en las batallas del Bajío que decidieron el curso de la contienda, coordinó años más tarde su primera campaña a la Presidencia de la República y sirvió en su gobierno como secretario de Comunicaciones y Obras Públicas.

De la casa del general Aguirre, en donde el retrato del general Obregón ocupaba un lugar prominente, habría de mudarse 
unos años después a una granja de Churubusco en la que un cuadro del general Porfirio Díaz presidía los días y las horas. La dueña de la casa, a la sazón su tía abuela y madrina, veneraba la memoria del presidente Díaz, mientras que guardaba muy malos recuerdos de la revolución mexicana. Desde entonces el pequeño Álvaro Matute comprendió que había varias interpretaciones sobre un mismo personaje o acontecimiento histórico y que el estudio de estas diferentes visiones es apasionante y constituye en sí mismo un objeto de estudio. Y por si necesitara un recordatorio de lo azarosa que había sido la historia de nuestro país, su casa se encontraba enfrente del exconvento de San Diego de Churubusco, testigo mudo de la guerra entre México y Estados Unidos y de la toma de la capital en 1847, que ya desde entonces era un museo que recordaba estos acontecimientos.

Álvaro Matute se convirtió en universitario cuando ingresó a estudiar el bachillerato al plantel 5 de la Escuela Nacional Preparatoria, en donde además de cursar ese nivel educativo participó de los años dorados del teatro preparatoriana bajo la dirección de Héctor Azar. Cuando llegó el momento de decidir la carrera que habría de estudiar, su elección inicial recayó en Ciencias Políticas, pero un año más tarde habría de pedir su cambio a la carrera que habría de abrazar desde entonces con singular pasión y en la que tanto habría de sobresalir: la licenciatura en Historia, en la Facultad de Filosofía y Letras de la entonces flamante Ciudad Universitaria de la UNAM.

Entre los maestros de la Facultad que ejercieron una importante influencia en Matute, ya fuera en el aula o en conferencias impartidas en aquellos años, encontramos una afortunada combinación de mexicanos que correspondían a la primera generación de historiadores académicos que, aunque provenían de otras disciplinas, principalmente del Derecho, acometieron la tarea de enseñar y escribir la historia con rigor intelectual, así como de españoles que formaban parte del exilio que con gran visión Lázaro Cárdenas acogió en nuestro país. Dentro de los 
primeros destacaban Edmundo O'Gorman, Ernesto de la Torre Villar, Eduardo Blanquel, Miguel León Portilla, Francisco de la Maza y Salvador Azuela, Josefina Vázquez entre otros. Entre los transterrados destaca Juan Antonio Ortega y Medina y José Gaos, profesor excátedra, dejó una profunda huella en él, así como la obra y el recuerdo de Ramón Iglesia.

Por aquellos años el joven Matute tuvo también una intensa vida cultural. Como hijo de dos actores que participaron de la época de oro del cine mexicano, era normal que se interesara por el cine desde joven. Durante sus años de juventud vivió con su madre, Estela Aguirre (mejor conocida en el ambiente artístico y cultural como Estela Matute), con quien concurría al teatro, la ópera y al amplio repertorio de actividades culturales de la ciudad de México de los años sesenta, cuando coexistín, no sin tensiones, las últimas manifestaciones del nacionalismo posrevolucionario con los afanes cosmopolitas de las nuevas generaciones. Fue en esos años cuando incursionó en el periodismo radiofónico, hablando de temas culturales en Radio uNAM. Enrique Krauze lo recuerda como redactor del programa "Los libros al día", que, en sus palabras, "tenía la virtud de ser vivaz sin ser frívolo, informado sin ser tedioso, claro sin ser superficial. Estaba hecho por un hombre que no leía las solapas de los libros: leía los libros". En aquel entonces se imaginaba al redactor del programa como un viejo erudito, pero años más tarde se sorprendería al conocerlo personalmente y constatar que era apenas unos años mayor que él. ${ }^{1}$

$\mathrm{Al}$ terminar sus estudios de licenciatura se incorporó como profesor en el Plantel 4 "Vidal Castañeda y Nájera" de la Escuela Nacional Preparatoria. A partir de 1971 sería profesor de Historiografía de México en el Colegio de Historia de la Facultad

${ }^{1}$ Respuesta de Enrique Krauze al discurso de ingreso de Álvaro Matute a la Academia Mexicana de la Historia. https://www.acadmexhistoria.org.mx/ pdfs/discursos/SILLON_11_ALVARO_MATUTE.pdf 
de Filosofía y Letras de la unAm. También sería profesor en la Facultad de Ciencias Políticas y Sociales y en el Centro de Enseñanza para Extranjeros de la Universidad Nacional, en el Centro de Estudios Históricos de El Colegio de México, en la Universidad Iberoamericana, la Escuela Nacional de Antropología e Historia, el Centro de Investigación y Docencia en Humanidades del Estado de Morelos, El Colegio de San Luis Potosí, el Instituto de Investigaciones Históricas de la Universidad Autónoma de Tamaulipas, la Universidad Autónoma de Baja California Sur, en la Universidad Juárez del Estado de Durango y la Universidad de Guanajuato. Álvaro Matute también impartió clases en la Universidad Complutense de Madrid y fue investigador visitante en El Colegio de México y en el Saint Anthony's College de la Universidad de Oxford.

En 1970 Matute se tituló como licenciado y ese mismo año recibió una beca para realizar estudios de posgrado en la Universidad de Texas en Austin, que interrumpió ante la oportunidad de ingresar como investigador al Instituto de Investigaciones Históricas de la unam, que desde entonces sería su casa. Esta circunstancia lo llevó a realizar sus estudios de maestría y doctorado en Historia en la Facultad de Filosofía y Letras de la UNAM. Las tesis con las que obtuvo ambos grados correspondieron a investigaciones que realizó dentro del proyecto de la Historia de la Revolución Mexicana, coordinado por Daniel Cosío Villegas y Luis González y González en El Colegio de México.

El proyecto de la Historia de la Revolución Mexicana le dio a Matute la oportunidad de ocuparse de uno de los periodos que más le interesaban desde la niñez. A él correspondió, en el plan original de la obra, la elaboración de tres libros: Las dificultades del nuevo Estado, La carrera del caudillo y El caudillo en el poder, trilogía que cubre el período que va desde el inicio del gobierno constitucional de Venustiano Carranza, en mayo de 1917, hasta el fin de la presidencia de Álvaro Obregón en noviembre de 1924. Las dificultades del nuevo Estado 
da cuenta del gobierno de Carranza y ofrece un análisis muy completo de la situación de México desde la toma de posesión del "varón de Cuatrociénegas" como presidente de la República hasta su muerte en Tlaxcalantongo. Su formato recuerda un poco La historia moderna de México, de don Daniel Cossío Villegas, sobre todo por la división entre la vida política interior y exterior, la economía y la sociedad. Por el contrario, La carrera del caudillo es una narración más libre del proceso que lleva a Álvaro Obregón a la presidencia de la República tras la caída de Carranza, así como del interinato de Adolfo de la Huerta, que a pesar de su brevedad contribuyó de manera decisiva a la pacificación del país.

Tuve el enorme honor y privilegio de colaborar con el doctor Matute en la elaboración de la última parte de la trilogía: $E l$ caudillo en el poder, que da cuenta de la presidencia de Âlvaro Obregón, cuatro años decisivos en la institucionalización de la política y en la construcción del presidencialismo mexicano. Además de los indiscutibles aciertos del presidente Obregón, como la creación de la Secretaría de Educación Pública y el apoyo inicial a su primer titular, José Vasconcelos, sigue la trama de la sucesión presidencial que dividió al grupo político sonorense y que desembocó en la rebelión delahuertista, así como las dificultades del reconocimiento externo y la compleja relación con la Iglesia, que habría de desembocar en el gobierno siguiente en el conflicto cristero.

Además de los tres grandes libros sobre el periodo 1917-1924, Matute incursionó en otros temas directa o indirectamente relacionados con la revolución mexicana. Destacan sus trabajos sobre Felipe Ángeles, la historia del Ejército revolucionario, el espionaje durante la Revolución y diversos aspectos de la historia cultural e intelectual, entre los que sobresalen los que tratan sobre el Ateneo de la Juventud y la política educativa de José Vasconcelos. Algunos de estos trabajos fueron reunidos en el libro La Revolución Mexicana: actores, escenarios, acciones, 
publicado originalmente por el Instituto Nacional de Estudios Históricos de la revolución mexicana (actualmente de las Revoluciones de México) en 1993 y que en una segunda edición fue coeditado por el Instituto Nacional de Estudios Históricos sobre la Revolución Mexicana y Editorial Oceano en 2002.

Pocos universitarios logran una combinación tan equilibrada entre la docencia, la investigación, la difusión de la Cultura y la participación institucional como fue el caso de Álvaro Matute Aguirre. Fue maestro de muchas generaciones en el Colegio de Historia de la Facultad de Filosofía y Letras, en el posgrado de Historia de la unam, en El Colegio de México y en otras instituciones nacionales y extranjeras. Director y cotutor de una gran cantidad de tesis de doctorado, sabía dirigir sin imponer, orientar sin inducir; en pocas palabras, formó a muchas generaciones respetando siempre las ideas, los intereses y las motivaciones de sus alumnos.

Su compromiso con la docencia fue total. Participó en las revisiones de los planes de estudio de la licenciatura de Historia de la Facultad de Filosofía y Letras y del Posgrado en Historia, así como en la creación de la especialización y la maestría en Historia de México que impartieron de manera conjunta los Institutos de Investigaciones Históricas de la UNAM y de la Universidad Autónoma de Tamaulipas y que fueron semilleros de un importante grupo de historiadores tamaulipecos. Su compilación para apoyar la docencia México en el siglo XIX: antología de fuentes e interpretaciones históricas es uno de los libros más reeditados de la UNAM.

Álvaro Matute escribió libros fundamentales para entender la historia de la revolución mexicana, la educación y la cultura en México, la institucionalización del Ejército y la política en México, la historiografía y la teoría y la filosofía de la historia. Su temprana compilación La teoría de la bistoria en México, publicada en 1974 en la colección Sepsetentas y recientemente reeditada por el Fondo de Cultura Económica, inauguró una línea de 
investigación en nuestro país en la que habría de profundizar, formando a varios investigadores que están en condiciones de perseverar en ella. Desde su tesis de licenciatura Lorenzo Boturini y el pensamiento histórico de Vico, publicada por el Instituto de Investigaciones Históricas en 1976, había mostrado su interés por la vinculación entre la teoría de la historia y la historiografía.

La historiografía fue sin lugar a dudas una de sus grandes pasiones. Lo mismo en los cursos de licenciatura que en los seminarios de posgrado fue un incansable promotor, junto con Evelia Trejo, su alumna, colega y esposa, del análisis historiográfico, con la firme convicción que constituye la columna vertebral del estudio de la historia. Sus contribuciones al análisis de la historiografía mexicana son muy numerosas, pero vale la pena recordar en este espacio Pensamiento historiográfico mexicano del siglo XX: la desintegración del positivismo (1911-1935), publicado por el Fondo de Cultura Económica en 1999 y Cuestiones de Historiografía Mexicana, editado por la Facultad de Filosofía y Letras y el Seminario de Cultura Mexicana en 2014.

También fue un incansable conferencista, presentador de libros y divulgador de la historia, siempre dispuesto a viajar a los lugares más recónditos del país para acercar a los estudiosos y al público en general al conocimiento histórico. A muy temprana edad coordinó el tomo dedicado a la revolución mexicana de la Historia de México de Editorial Salvat. Desde entonces no escatimó ni tiempo ni esfuerzo para divulgar la historia. Como miembro del Seminario de Cultura Mexicana recorrió el país atendiendo numerosas invitaciones para acercar a la población en general el conocimiento histórico.

Sirvió a la Universidad no solo a través de sus tres funciones más importantes, sino como funcionario y miembro de múltiples cuerpos colegiados y jurados. Fue subdirector en la Dirección General de Asuntos del Personal Académico, Director del Centro de Enseñanza para Extranjeros y del Centro de Estudios sobre los Estados Unidos de Norteamérica y coordinador 
del Posgrado en Historia de la Facultad de Filosofía y Letras, consejero universitario y miembro de la Junta de Gobierno en momentos decisivos para la Universidad.

Pero además de su impresionante curriculum, que le valió múltiples reconocimientos en vida, como el Premio Nacional de Ciencias y Artes, el Premio Universidad Nacional, el emeritazgo como investigador del Instituto de Investigaciones Históricas y como miembro del Sistema Nacional de Investigadores, así como su ingreso al Seminario de Cultura Mexicana y a la Academia Mexicana de la Historia y a la Academia Mexicana de la Lengua, tuvo el don de tocar con su sabiduría y su generosidad la vida de muchas personas que gracias a él, somos mejores historiadores y universitarios. Que descanse en paz y se mantenga vivo por siempre en el afecto de su familia, sus amigos y sus muchos discípulos, así como en la vigencia de su vasta obra. 